\title{
TREATMENT OF LUBRICATING OIL USED WITH THE USE OF SOLVENTS AND ADSORBENT MATERIALS
}

\author{
ROSA, Mirna Sales Loiola ${ }^{1^{*}}$; SILVA, Ives Brian Campelo Leite ${ }^{1}$; ARAÚJO, Neyzon Talles \\ Matos $^{1}$; FIGUEIREDO, Francisco Cardoso ${ }^{1}$; SANTOS JUNIOR, Jose Ribeiro dos ${ }^{1}$ \\ ${ }^{1}$ Universidade Federal do Piauí, Centro de Ciências da Natureza,Campus Universitário Ministro Petrônio \\ Portella - Bairro Ininga -, cep 64049-550, Teresina, Piauí, Brasil \\ (fone: +55 86 3215-5525; fax: +55 863215-5526) \\ e-mail:mirnasales01@hotmail.com
}

Received 09 December 2017; received in revised form 07 February 2018; accepted 07 February 2018

\section{RESUMO}

O desenvolvimento das atividades humanas nos setores industriais e de transportes tem destinado os óleos lubrificantes usados e contaminados (OLUC) em aterros sanitários, águas superficiais e subterrâneas. Outra alternativa é a incineração, que libera altas concentrações de HPA's (hidrocarbonetos policíclicos aromáticos), dioxinas na atmosfera e o rerrefino. Dentre essas destinações, a única adequada é o rerrefinamento que recupera as propriedades iniciais e reutiliza o óleo. Com base na literatura, o processo de rerrefinamento utilizando as etapas de extração e adsorção é eficaz, de baixo custo, tornando o produto apto a entrar na cadeia produtiva novamente. Então, esta revisão destaca o processo de recuperação do óleo base, a partir da extração com solvente e materiais adsorventes e as caracterizações do óleo novo, OLUC e óleo recuperado por espectroscopia na região do infravermelho por Transformada de Fourier (FTIR) e análise térmica.

Palavras-chave: OLUC, rerrefinamento, extração e adsorção.

\section{ABSTRACT}

The development of human activities in the industrial and transportat sectors has increased the contamination of water bodies by the release of used and contaminated lubricating oils used and contaminated (OLUC). To contain such contaminations, the process of re-refining the OLUC has been used worldwide to recover the base oil. Based on the literature, this process using the extraction and adsorption steps is effective, low cost, making the product able to enter the production chain again. So, this review highlights the recovery process of the base oil, from the extraction with solvent and adsorbent materials and the characterizations of the new oil, OLUC and oil recovered by Fourier Transform Infrared Spectroscopy (FTIR) and thermal analysis.

Keywords: OLUC, re-refining, extraction and adsorption. 


\section{INTRODUÇÃO}

O aumento populacional vem impulsionando o desenvolvimento descontrolado das múltiplas atividades humanas nos setores industriais e de transportes e consequente utilização de óleos lubrificantes seja ele de base mineral ou sintética (GAVRILESCU et al., 2015; ZHANG et al., 2016; SENAPATI et al, 2000).

O óleo lubrificante é o fluido mais importante para todos os tipos de veículos e máquinas, antecedido apenas dos combustíveis (BRESSANI et al, 2006; TSAI, 2011). Estima-se que o Brasil utiliza cerca de $1.520 .000 \mathrm{~m}^{3}$ de óleo lubrificante por ano, como consequência da industrialização do país e da elevada frota de veículos (SILVEIRA et al, 2010). O seu componente principal é o óleo base refinado a partir de petróleo bruto ou sintetizado em laboratório, onde suas características são controladas misturando-o á aditivos, tais como, partículas metálicas ( $\mathrm{Zn}, \mathrm{Cd}, \mathrm{Cr}, \mathrm{Pb}, \mathrm{Fe})$, polímeros poli-alfa-olefinas (PRIOLA et al, 1985), poli-isobuteno, dentre outros, para neutralizar os ácidos gerados durante a combustão e reduzir a reação de oxidação (SILVEIRA et al, 2006; MELO e SOUZA, 2015; HU et al, 2017).

No entanto, o lubrificante sofre várias alterações por degradação e contaminação após certo tempo de uso recomendado pelos fabricantes, como por exemplo, produção de ácidos orgânicos (YANG et al, 2016), cetonas (YAO et al, 2016), compostos aromáticos polinucleares de viscosidade elevada e potencialmente cancerígenos (OSMAN; SAYED e TAMAN, 2017) e com isso há a redução do desempenho de máquinas e motores (THOENES, 1975).

Uma vez não usados são descartados muitas vezes de forma inadequada em corpos hídricos, gerando graves danos ambientais, pois o óleo produz uma barreira na parte sobrenadante, impedindo a oxigenação e a passagem dos raios solares e, consequentemente, esgotando a capacidade de fotossíntese e sobrevivência dos seres aeróbios (SINGH et al, 1997). Além disso, podem ser queimados de forma não controlada, liberando altas concentrações de HPA's (hidrocarbonetos policíclicos aromáticos) e dioxinas na atmosfera, altamente tóxicos e cancerígenos (KUCZENSKlet al, 2014; WILLING, 2001).
Para evitar tais contaminações, a única destinação adequada dos óleos lubrificantes usados é a regeneração das propriedades iniciais e reutilização por rerrefino (HAMAD; et al., 2005; KULKARNI e RATHOD, 2014).

Segundo o artigo 3 da resolução número 362/05 do Conselho Nacional de Meio Ambiente (CONAMA, 2005), o rerrefinamento trata-se da categoria de processos industriais de remoção de contaminantes, produtos de degradação e aditivos dos óleos lubrificantes usados ou contaminados (OLUC), conferindo aos mesmos características de óleos básicos, conforme legislação específica (SRIVASTAVA, 2014). Este processo vem sendo muito utilizado por fabricantes de aditivos e formuladores desse tipo de óleo devido à diminuição das reservas mundiais de petróleo e preocupações ambientais (LUNA et al, 2011; KHAN et al, 2016).

No entanto, o processo de rerrefinamento utilizado pelas indústrias são muito demorados, utilizam bastante energia e reagentes nas etapas de homogeneização, caracterização e segregação das amostras de óleo usado, desidratação e obtenção de hidrocarbonetos leves, tratamento químico ou extração, recuperação dos solventes/catalisadores e clarificação do óleo lubrificante contaminado (BU et al, 2013; CARRETEIRO e MOURA, 1975; DHABHAl et al, 2016; KUCZENSKI et al, 2014; LAM; RUSSELL; CHASE; 2010).

A fim de reduzir a quantidade de energia e de reagentes nas etapas do processo de tratamento do óleo lubrificante, pode-se utilizar a extração com solventes orgânicos com até quatro átomos de carbonos, como, acetona, butanol (HAMAD, AL-ZUBAIDY e FAYED, 2005), acetato de etila, dentre outros (MOHAMMED et al, 2013; COSTA et al, 2010) que precipitam os contaminantes. Entretanto, o processo de extração por si só não é tão eficaz no processo de remoção dos contaminantes do óleo (CASTRO e CASTRO, 2010). Logo, também é utilizado o processo de adsorção por materiais adsorventes locais, tais como, argilas ácidas (SALEM, SALEM e BABAEI, 2015), carvão ativado (CHTOUROU, FRIKHA e TRABELSI, 2006) e celulose (YINet al, 2017) para adsorver corantes dissolvidos nos OLUC's garantindo o clareamento do óleo e um atrativo para ser comercializado (ALI, RIPIN e AHMAD, 2010; FILHO et al, 2010). 
Com base nisso, o objetivo principal desta revisão é apresentar e discutir os artigos mais recentes que utilizam solventes e materiais adsorventes naturais no processo de rerrefinamento de OLUC e a caracterização dos óleos lubrificantes.

\section{DESENVOLVIMENTO OU MATERIAL MÉTODOS OU PARTE EXPERIMENTAL}

\section{CONTAMINANTES DO OLUC}

O OLUC é um produto perigoso que contém contaminantes, tais como, metais tóxicos como o chumbo e cádmio e metais de aditivação como cálcio e zinco (MELO e SOUZA, 2015; ROCHER et al, 2004). Além disso, ficam contaminados com água, partículas sólidas, poeira e resíduos de desgaste, combustíveis e frações de combustível, carbono, fuligem, lama, glicol, componentes de nitração e de sulfatação (WAGNER; LUTHER e MANG, 2001).

É importante ressaltar que à medida que aumenta a distância percorrida pelo veículo, também aumenta a concentração de radicais livres e a sua taxa de degradação. Logo, os aditivos são degradados, a partir de cerca de $6000 \mathrm{~km}$ percorridos pelo veículo (SRIVASTAVA, 2014).

Os aditivos misturados aos óleos lubrificantes básicos para formar 0 óleo lubrificante acabado, segundo a Petrobrás (2017), são: dispersantes, succinimidas polialquênicas, polímeros de hidrocarbonetos contendo grupos polares, polimetacrilatos contendo nitrogênio, sulfonato de metal sódio, magnésio, cálcio ou bário, (SRIVASTAVA, 2014) detergentes sulfonados, salicilatos e fenatos de metal sódio, magnésio, cálcio ou bário (MANGAS, SOGORB e VILA NOVA, 2014) detergentes alcalinos (sulfonados, salicilatos e fenatos de metal magnésio, cálcio ou bário), antioxidantes, ditiofosfato de zinco, fenóis e aminas aromáticas, sulfetos orgânicos, fosfitos orgânicos, derivados orgânicos de cobre (RASBERGER, 1997), passivadores de metais (ditiofosfato de zinco, sulfatos orgânicos, fenatos metálicos, compostos orgânicos nitrogenados), antiespumantes (polímeros do silicone, polimetacrilatos), anticorrosivos (ditiofosfato de zinco, detergentes alcalinos, ditiocarbamatos metálicos, terpenos fosforados ou sulfurados, ácidos succínicos de alquenila, fenóis de alquila propoxilatos ou etoxilatos, imidazolinas), antiferrugem (sulfonados de metal, ácidos graxos, amina), agentes de oleosidade (ácido oleico e óleo de banha), agentes antidesgaste, como por exemplo, ditiofosfato de zinco, álcool ditiocarbamatos, tri-cresil-fosfato, derivados orgânicos de cálcio, magnésio, zinco, cádmio, níquel, vanádio, selênio (COLEMAN,2003), modificadores de friç̧ão (bi-sulfeto de molibdênio, grafite), agentes de adesividade (poliisobutilenos, asfalto), emulsificantes (breu, sabões de ácidos graxos, ácidos sulfônicos, ácidos naftênicos, sulfonados de sódio), biocidas (compostos de formaldeídos, derivados orgânicos de cloro e mercúrio), demulsificantes (sulfonados de bário, cálcio e zinco), abaixadores de ponto de fluidez (polimetacrilatos, polialquiamidas, naftalenos alquilados, polímeros de copolímeros de alfa-olefinas), melhoradores de índice de viscosidade (copolímeros de olefinas, copolímeros de estireno-dieno hidrogenado, polialquil metacrilatos), corantes, compostos orgânicos nitrogenados, tais como, anilinas e azocompostos (CHEN et al, 2004; TAGAWA et al, 2012).

Dentre os aditivos, os compostos orgânicos produzem contaminantes quando são expostos a altas temperaturas e pressões, pois sofrem oxidação na presença de oxigênio (SANTOS et al, 2004; RASBERGER, 1997; GATTO et al, 2007). Os contaminantes formados são os óxidos de nitrogênio ( $\mathrm{NO}, \mathrm{NO}_{2}$ e $\mathrm{N}_{2} \mathrm{O}_{4}$ ) que reagem com a água e produzem ácidos de elevado peso molecular formando uma lama ou borra ácida que precipita (PARENAGO e BAKUMIN, 1997; EMAM e SHOAIB, 2011).

Os óxidos de enxofre, tais como, $\mathrm{SO}_{2}$ e $\mathrm{SO}_{3}$ são subprodutos da oxidação do enxofre em combustível que são formados durante a combustão bem como a oxidação de aditivo (SHIUNG; RUSSELL e CHASE, 2010). Estes compostos reagem com a água formada durante a combustão e produz ácidos inorgânicos, como por exemplo, o ácido sulfúrico, aumentando a produção de borra ácida no óleo degradado (SUEN; WARD e MILLER, 2012).

Os produtos de oxidação dos compostos orgânicos de óleo de motor também correspondem à formação de materiais oxigenados, tais como, compostos hidroxila, principalmente de ácidos carboxílicos, compostos carbono-oxigênio que inclui grupos carbonila, hidroxi e compostos de éter (PAWLAK; RAUCKYTE e OLOYEDE, 2008; JHA, 2005; 
ZZEYANI et al, 2017). Como também, a formação de fuligem durante uma reação de queima entre a mistura de combustível e ar, alterando a viscosidade do óleo (SRIVASTAVA, 2009; BOUGHTON e HORVATH, 2004).

\section{RERREFINAMENTO POR DESTILAÇÃO}

A tecnologia de recuperação do óleo base (rerrefinamento) começou em 1935, mas somente nos últimos 20 anos, tecnologias de rerrefinação foram desenvolvidas (WAGNER, LUTHER e MANG, 2001). Este processo é a melhor opção para a recuperação do óleo útil a partir do óleo usado descartado, uma vez que recupera os hidrocarbonetos que não sofreram com processos de degradação e elimina os contaminantes do OLUC (SANTOS, 2011).

Segundo, Guimarães (2006), o processo de rerrefinamento utilizado no Brasil estão basicamente relacionados a operações de destilação e tratamentos químicos pela adição de ácido sulfúrico e argilas ácidas, fortemente redutores para promover a remoção de corantes do OLUC (CAREY, 2013; MENZEL; LINDNER; NIRCHL, 2012). A desvantagem do processo de rerrefinamento realizado pelas industriais brasileiras está na exigência de diversas etapas, além disso, na produção de borra ácida, fazendo com que a obtenção do produto desejado seja demorada (SONG et al, 2010).

\section{RERREFINAMENTO POR TECNOLOGIA ULTRASSOM}

A tecnologia de ultrasson no processo de rerrefinamento de OLUC acontece apenas com a utilização de solventes e ondas ultrassônicas. A incorporação do solvente ao óleo lubrificante usado é feita em banho termostatizado e a extração do óleo acontece sob ondas ultrassônicas de baixa frequência $(37$ e $80 \mathrm{KHz})$ em banho de ultrassom (SOUZA, 2015; POVEDANO e CASTRO, 2013).

\section{RERREFINAMENTO POR EXTRAÇÃO DE SOLVENTE COM MEMBRANA}

De acordo com Yoshiie (2012), a recuperação de óleo base do OLUC pode ser realizado por extração de solvente com membrana de borracha. Neste processo, o solvente orgânico é circulado em circuito fechado, levando a uma economia de recursos
(GUIMARÃES, 2006). O resíduo consiste apenas em impurezas no óleo lubrificante de resíduos, tais como partículas suspensas, gotículas aquosas, fuligem e alcatrão, levando a uma redução de resíduos. Este processo também inclui uma destilação fracional inferior a $100^{\circ} \mathrm{C}$ para separar o solvente orgânico dos óleos.O filme de borracha é utilizado como membrana, cuja estrutura de rede de polímero funciona como filtro para separar as impurezas do óleo dissolvido em solvente (AKIMOTO; IIDA; SATO, 2007).

\section{RERREFINAMENTO POR MISTURA DE SOLVENTES POLARES}

Rincon; Canizares; Garcia (2005), propuseram um método eficaz de recuperar óleo base a partir de OLUC é com a utilização da mistura dos solventes polares: metiletilcetona (MEK) e 2-propanol com uma proporção 2propanol/MEK de $3 \mathrm{~g} / \mathrm{g}$. No entanto, somente esta mistura de solventes ainda não conseguiu remover completamente os metais e os produtos de oxidação, sendo necessário também a adição de pequenas quantidades de $\mathrm{KOH}$ (de 1 a $7 \mathrm{~g} / \mathrm{kg}$ de solvente).

\section{RERREFINAMENTO POR ÁCIDO ACÉTICO}

De acordo com Hamawand; Yusaf; Rafat (2013), a reciclagem de óleos de motores usados pode ser realizada com ácido acético. Esse processo fornece ao óleo reciclado o potencial para ser reutilizado nos motores dos carros depois de adicionar os aditivos necessários. A vantagem de usar o ácido acético é que ele não reage ou reage apenas ligeiramente com os óleos de base. Após a adição de 0,8\% em volume de ácido acético ao óleo usado, foram separadas duas camadas, um óleo transparente de cor vermelha escura e uma lodo escuro preto na parte inferior do recipiente (NING et al, 2009).

\section{RERREFINAMENTO POR SOLVENTES E MATERIAIS ADSORVENTES}

O solvente é usado na etapa de extração do processo de rerrefinamento do OLUC, para separar os aditivos e subprodutos de envelhecimento que contaminam 0 óleo (ZUBAIDY e ABOUELNASR, 2010). Uma vez realizada, não é necessário o tratamento químico por ácido sulfúrico. Entretanto, o processo de extração não retém a cor e o odor dos 
contaminantes do óleo, sendo necessária também no processo a utilização de materiais adsorventes (MORAIS et al, 2013; PATRICIO, HOTZA e JÚNIOR, 2014; SANTOS, 2011).

Boa parte dos artigos revisados utilizou vários solventes isolados ou misturados em diversas proporções com o óleo contaminado para determinar o rendimento do óleo base e em seguida fez o uso de materiais adsorventes, como pode ser visto na tabela 1 .

$\mathrm{Na}$ Tabela 1, observa-se que o melhor rendimento de óleo base foi na proporção 3:1 de solvente para óleo. Além disso, segundo os estudos de MOHAMMED et al, 2013, MARTINS et al, 2012 e DURRANI,PANHWAR e KAZI, 2011, o melhor processo de extração foi utilizando 0 solvente 1-butanol e para 0 acabamento do óleo lubrificante o uso de argilas ativadas com ácido (GHOUTI e ATOUM, 2009).

Boa parte dos artigos revisados utilizaram as argilas do grupo das esmectitas (montmorilonita, beidelita, bentonita, dentre outros) na forma natural e ativada com ácido. Esse grupo de argilas é conhecido como "argilas clareadoras" ou "argilas adsorventes", devido à sua estrutura lamelar, que proporciona elevadas áreas superficiais específicas (ERRAISet al, 2011; SALEM; SALEM e BABAEI, 2015). Uma vez tratada com ácido, aumenta a eficiência do processo de adsorção de corantes.

A fórmula estrutural das esmectitas é do tipo, 2:1, isto é, são constituídos por duas folhas ou lamelas tetraédricas de silicato, e uma lamela central octaédrica do tipo Gibbsita $\left(\mathrm{Al}_{2}(\mathrm{OH})_{6}\right)$ e pode ser representada conforme a figura 1 .

No processo de rerrefinamento, $\mathrm{o} \mathrm{pH}$ do adsorvente, afeta a adsorção da cor e odor do óleo contaminado. A maioria dos estudos da Tabela 3 mostrou que a quantidade de adsorção é maior em argilas ativadas com ácido do que as argilas naturais. Isso acontece devido a protonação dos grupos hidroxila $\left(\mathrm{OH}^{+1}\right)$ da superfície do material adsorvente em meio ácido, pois a superfície protonada garante o processo de adsorção física por atração eletrostática com o contaminante; Já os grupos desprotonados $\left(\mathrm{OH}^{-1}\right)$ garantem adsorção química por ligação covalente e/ou de hidrogênio com o contaminante. O mecanismo de adsorção por atração eletrostática entre a superfície adsorvente $\left(\mathrm{S}^{+}\right)$e 0 corante $\left(\mathrm{R}-\mathrm{SO}_{3}^{-1}\right)$ contaminante do óleo mineral pode ser ilustrado conforme a Equação 1. (MITROPOULOS et al, 2012):

$$
\mathrm{S}^{+}+\mathrm{R}-\mathrm{SO}_{3}^{-} \rightarrow \mathrm{S}^{+-} \mathrm{SO}_{3}-\mathrm{R}
$$

Uma vez tratado, o OLUC possui todas as características necessárias para ser aditivado e voltar a ser comercializado (WILING 2001). Entretanto, a verificação das características dos óleos básicos far-se-á mediante o emprego das Normas Brasileiras Registradas-NBR e dos métodos da American Society for Testing and Materials - ASTM (SIMEPETRO, 2017).

$\mathrm{Na}$ Tabela 2, apresentam-se os resultados das caracterizações físicas e químicas, tais como, cor, viscosidade cinemática (cSt) a $40^{\circ} \mathrm{C}$ (Visc. $40^{\circ} \mathrm{O}$ ), viscosidade cinemática (cSt) a $100^{\circ} \mathrm{C}$ (Visc. $\left.100^{\circ} \mathrm{C}\right)$ e teor de cinzas (\% cinzas) dos óleos recuperados. Encontrados nos artigos referenciados e sua classificação segundo a Portaria ANP130, 1999.

A partir da Tabela 2, verificou-se que os óleos recuperados com solvente polar e materiais adsorventes nos artigos de Mohammed et al, 2013; Salem, Salem, Babaei, 2015; Zaky e Tawfik, 2011; Emam e Shoaib, 2013, são classificados em neutro pesado $R R$, neutro médio, neutro leve e neutro pesado RR, respectivamente.

\section{CARACTERIZAÇÃO}

As técnicas de caracterização, tais como, infravermelho por Transformada de Fourier (FTIR), RMN ${ }^{13} \mathrm{C}$ e análise térmica (termogravimetria e calorimetria), indicam os produtos da reação de oxidação e degradação térmica nas amostras de óleo novo, OLUC e óleo recuperado (OLIVEIRA et al, 2004).

Dentre os artigos revisados, apenas o trabalho de DINIZ, ALMEIDA e SANTOS, 2009, caracterizaram as amostras de óleos, conforme a Tabela 3.

No trabalho de DINIZ, ALMEIDA e SANTOS, 2009, eles propuseram que no espectro do óleo novo, OLUC e óleo recuperado a região em $723 \mathrm{~cm}^{-1}, 821 \mathrm{~cm}^{-1}$ e $1158 \mathrm{~cm}^{-1}$ correspondemadeformação $\mathrm{C}-\mathrm{H}$ para os grupos $\mathrm{CH}_{2}$ e $\mathrm{CH}_{3}$; Jáem $1378 \mathrm{~cm}^{-1}$ e $1470 \mathrm{~cm}^{-1}$ correspondem ao aparecimento de compostos 
orgânicos oxigenados no OLUC, em $1600 \mathrm{~cm}^{-1}$ indicam a provável ligação $\mathrm{C}-\mathrm{C}$ no óleo novo, OLUC e óleo recuperado, em $1705 \mathrm{~cm}^{-1}$ sugere a deformação axial de C-O de álcoois no OLUC, consoante a Tabela 4.

A degradação da amostra precisa ser complementada por outras técnicas que identificam a degradação da amostra, como por exemplo, a análise térmica por termogravimetria (BURGUERA et al, 2005).

A termogravimetria é uma técnica de caracterização que determina a massa da amostra em função da temperatura ou tempo (SANTOS et al, 2013); Já a termogravimetria derivada (DTG), consiste da derivada da variação de massa em relação ao tempo (dm/dt) que também é registrada em função da temperatura ou tempo.

O trabalho de DINIZ, ALMEIDA e SANTOS, 2009, utilizaram a TGA/DTG para analisar a estabilidade térmica para estudar o perfil da decomposição e estabilidade térmica do óleo lubrificante usado em relação aos óleos recuperado e novo. $O$ processo de decomposição térmica do óleo novo, OLUC e óleo recuperado com 1-butanol teve início na temperatura de $126,18{ }^{\circ} \mathrm{C}, 45,83^{\circ} \mathrm{C}, 35,24$, respectivamente. Segundo HSU (2005), uma vez que o óleo novo apresentou a maior temperatura de início de degradação térmica em condições inertes, ele obteve a maior estabilidade em relação aos óleos usado e recuperado (GUAN et al, 2011).

\section{CONCLUSÕES}

Os estudos das literaturas mostraram que com o aumento populacional do planeta, houve um aumento na utilização de óleo lubrificante por ano e com isso o seu tratamento inadequado por queimadas descontroladas, liberando altas concentrações de HPA's (hidrocarbonetos policíclicos aromáticos) e dioxinas na atmosfera, altamente tóxicos e cancerígenos.

Para conter esses poluentes todos os artigos revisados, utilizaram 0 processo de rerrefinamento por extração utilizando solventes e/ou adsorção utilizando materiais adsorventes naturais e/ou ativados, não tóxicos, de baixo custo, facilmente disponíveis na natureza. Além disso, boa parte dos resultados mostrou que a melhor proporção de solvente para óleo na obtenção do óleo base foi a de 3:1 e que o acabamento do óleo base lubrificante depende de materiais adsorventes. Estes resultados foram corroborados em alguns artigos pelas técnicas de caracterizações dos óleos por FTIR e TGA/DTG.

\section{REFERÊNCIAS:}

1. Ali, W. M. S., Ripin, A \& Ahmad, A. Adsorption of heavy metal from recovered base oil using zeolite. Journal of Applied Sciences, 2010,10, 2688-2692.

2. Akimoto, M., lida, $T$ \&Sato, S. "Hydrothermal Dechlorination of Reclaimed Oil in a Continuous Packed-Bed Reactor", Journal of Chemical Engineering of Japan, 2007, 40, 874-878.

3. Angulo, J. Regeneracion de Aceites Usados por Extraccion con Propano. Ing. Quım. 2002, 153.

4. Ashcroft, J. M., Hartman, K. B.,Mackeyevy, Hofmann, C., Pheasant, S. Alemany, L. B \& Wilson, L. J. Functionalization of individual ultra-short single-walled carbon nanotubes. Nanotechnology, 2006, 17, 5033 - 5037.

5. Boughton, B \& Horvath, A. Environmental assessment of used oil management methods. Environ Sci Technol, v. 38, n. 2, p. 353-358, 2004.

6. Bressani, F. A., Silva, H. O., Nóbrega, J. A. Costa, L. M \& Nogueira, A. R. A. Digestão de óleo lubrificante encapsulado em forno de microondas com radiação focalizada por adição de amostra ao reagente pré-aquecido. Química Nova, 2006, 29, 1210-1214.

7. $\mathrm{Bu}, \mathrm{D}$. et al. Remediation of Used Motor Engine Oil Contaminated Soil: A Soil Washing Treatment Approach. Civil \&Environmental Engineering, 2013, 3.

8. Burguera, J. L., Burguera, M., Anton, R. E \& Carrero, P. Determination of aluminum by electrothermal atomic absorption spectroscopy in lubricating oils emulsified in a sequential injection analysis system. Talanta, 2005, 68, 179-186.

9. Carey, J. T., Galiano-roth, A. S., Wu, M. M \& Haigh, H. M. Low sulfur and low metal additive formulations for high performance industrial oils. US patent 8,394,746, 2013, March 12.

10. Carreteiro, R.P \& Moura, C.R.S. Lubrificantes e Lubrificação. São Paulo: L.T.C. Editora S.A, 1975, p.493.

11. Castro, R \& Castro, G. D. M. Gerenciamento do óleo lubrificante usado para a logística reversa: uma análise nos postos de combustíveis na cidade de pederneiras. XXX ENCONTRO NACIONAL DE ENGENHARIA DE PRODUÇÃO.2010, SP, Brasil. 
12. Chtourou, M., Frikha, H. M \& Trabelsi, $M$. Modified smectitic Tunisian clays used in the formulation of high performance lubricating greases. Applied Clay Science,2006, 32, 210216.

13. Chen, C. S., Chen, X. H., Liu, T. G., Yang, D., Zhang, G \& Yi, G. J. Chemical modification of carbon nano-tubes and tribological properties as lubricant additive. Acta Chim Sin,2004, 62, 1367-1372.

14. Coleman, K.S., Bailey, S.R., Fogden, S \& Green, M. L. H. Functionalization of singlewalled carbon nanotubes via the bingel reaction. Journal of the American Chemical Society, 2003, 125, $8722-8723$.

15. Conama, Resolução $n^{\circ} 362$, de 23 de junho de 2005. Ministério do Meio Ambiente, 2005.

16. Costa, J, et al. Monitoring genetically modified soybean along the industrial soybean oil extraction and refining processes by polymerase chain reaction techniques. Food Research International,2010, 43,301-306.

17. Ghouti, M. A \& Atoum, L. Virgin and recycled engine oil differentiation: A spectroscopic study. Journal of Environmental Management,2009, 90, 187-195.

18. Guan, L., Feng, X. L., Xiong, G \& Xie, J. A. Application of dielectric spectroscopy for engine lubricating oil degradation monitoring. Sensors and Actuators A: Physical, 2011, 168,22-29.

19. Guimarães, J. Rerrefino de óleos lubrificantes de motores de combustão interna pelo processo de ultrafiltração e adsorção. Dissertação (Mestrado em Engenharia Ambiental) - Programa de Pós graduação em Engenharia Ambiental da Universidade do Estado do Rio de Janeiro, Rio de Janeiro, 2006.

20. Durrani,H. A., Panhwar,M. I \& Kazi, R. A. ReRefining of Waste Lubricating Oil by Solvent Extraction. Mehran university research journal of engineering \& technology,2011, 30, 237246.

21. Diniz, P. I., Almeida, A \& Santos, J. C. O. Recuperação de óleos lubrificantes usados usando solvente polar: 1-butanol. VI Congresso de Iniciação Científica de Campina Grande, 2009.

22. Errais, E. et al. Efficient anionic dye adsorption on natural untreated clay: Kinetic study and thermodynamic parameters. Desalination,2011, 275, 74-81.

23. Emam, A. E \& Shoaib, M. A. Re-refining of used lube oil, by solvent extraction and vacuum distillation followed by hydrotreating. Petroleum \& Coal,2013, 55, 179-187.

24. Filho, A. L. J., Moura, M. G. L \& Ramos, S. C. A. Liquid-liquid Extraction and Adsorption on Solid Surfaces Applied to Used Lubricant Oils
Recovery. Brazilian Journal of Chemical Engineering, 2010, 27, 687 - 697.

25. Gatto, V. J., Elnagar, H.Y., Moehle, W. E \& Schneller, E. R. Redesigning alkylated diphenylamine antioxidant for modern lubricant. Lubrication Science,2007, 19, 2540.

26. Hamad, A., AL-zubaidy, E \& Fayed, M. Used lubricating oil recycling using hydrocarbon solvents.Journal of Environmental Management,2005, 74, 153-159.

27. Higgins, I. J., Gilbert, P. D \& Wyatt, J. Microbial degradation of oil in environment. Studies Environmental Science,1981, 9, 85.

28. Hsu, S. M. Boundary lubricating films: formation and lubrication mechanism. Tribology International,2005, 38, 305-312.

29. $\mathrm{Hu}, \mathrm{J}$. et al. Microscopic investigation on the adsorption of lubrication oil on microplastics. Journal of Molecular Liquids,2017, 227, 35135.

30. Gavrilescu, M., Demnerova, K.; Aamand, J., Agathos, J \& Fava, F. Emerging pollutants in the environment: present and future challenges in biomonitoring, ecological risks and bioremediation. New Biotechnology,2015, 32, 147-156.

31. Guimarães, J. Refino de óleos lubrificantes de motores de combustão interna pelo processo de ultrafiltração e adsorção. 2006. 95p. Dissertação (Mestrado) - Departamento de Engenharia Sanitária e do Meio Ambiente Universidade do Rio de Janeiro, Rio de Janeiro, 2006.

32. Jamil, M. S., ALI, W. M., Ripin, A \& Ahmad, A. Metals removal from recovered base oil using chitosan biopolymers. Journal of Applied Sciences,2010, 21, 2725-2728.

33. Jha, M. K. Re-refining of used lube oils: an intelligent and eco-friendly option. Indian Chem Eng,2005, 47, 209-211.

34. Khan, R. et al. Production of diesel-like fuel from spent engine oil by catalytic pyrolysis over natural magnetite. Journal of Analytical and Applied Pyrolysis,2016, 120, 493-500.

35. Kuczenski, B et al. Material flow analysis of lubricating oil use in California. Resources, Conservation and Recycling,2014, 93, 59-66.

36. Lam, S. S., Russell, A. D \& Chase, H. A. Microwave pyrolysis, a novel process for recycling waste automotive engine oil. Energy, 2010, 35, 2985-2991.

37. Luna, F. M. T. et al. Assessment of biodegradability and oxidation stability of mineral, vegetable and synthetic oil samples. Elsevier, Industrial Crops and Products,2011, 33, 579-583.

38. Mangas, I., Sogorb, M. A \& Vilanova, E. Lubricating oil. Encyclopedia of Toxicology (Third Edition),2014, 3, 670-676. 
39. Martins, S. J. et al. Recovery of lubricants used in diesel engines using polar solvents: 1butanol and 2-propanol. IX Congresso de Iniciação Científica da Universidade Federal de Campina Grande,2012.

40. Melo, M. L. S \& Souza, J. R. de. Estudo do efeito de matriz na determinação de metais em óleos básicos rerrefinados por fluorescência de raios $x$ por energia dispersiva. Quím. Nova, 2015, 38, 614-621.

41. Menzel, K., Lindner, J \& Nirchl, H. Removal of magnetite particles and lubricant contamination from viscous oil by HighGradient Magnetic Separation technique. Separation and Purification Technology, 2012, 92, 122-127.

42. Mohammed, R. R.et al. Waste lubricating oil treatment by extraction and adsorption. Chemical Engineering Journal, 2013, 220 , 343-351.

43. Morais, V. S. et al. Cor ASTM: um método simples e rápido para determinar a qualidade do biodiesel produzido a partir de óleos residuais de fritura. Quím. Nova,2013, 36, 587-592.

44. Mitropoulos, C. A., Lazaridis, K. N \& Kyzas, Z. $G$. Removal of dyes from aqueous solutions with untreated coffee residues as potential low-cost adsorbents: Equilibrium, reuse and thermodynamic approach. Chemical Engineering Journal,2012, 189 - 190, 148 159.

45. Ning, X., Wenxiang, W., Pingfang, $H$ \& Xiaoping, L. Effects of ultrasound on oily sludge deoiling. Journal of Harzardous Materials, 2009, 171, $914-917$.

46. Oliveira, J. C., Garcia, I. M., Gouveia, A., Vitor, E., Fernandes, V. J \&Silva, A. J. Thermoanalytical and Rheological Characterization of Automotive Mineral Lubricants after Thermal Degradation. Fuel, 2004, 83, 2393.

47. Osman, D. I., Sayed, K. A \& Taman, R. A.Recycling of used engine oil by different solvent. Egyptian Journal of Petroleum xxx, 2017.

48. Parenago, O. P \& Bakumin, V. N. Problem of inhibiting the high temperature oxidation of hydrocarbon, additive 97. Proceedings of Hungarian Chemical Society,1997, 81-88.

49. Pasila, A. A biological oil adsorption filter. Marine Pollution Bulletin,2004, 49, 10061012.

50. Patricio, S. J., Hotza, D \& Júnior, N. A. Adsorbent clays applied in vegetable oil clarification. Cerâmica,2014, 60, 171-178.

51. Pavia, L. D., Lampman, M, G., Kriz, S. G \& Vyvyan, R. J.Introdução à espectroscopia. $4^{\mathrm{a}}$ ed. Cengage Learning, 2010.

52. Pawlak, Z., Rauckyte, T \&Oloyede, A. Oil, grease and used petroleum oil management and environmental economic issues. Achiv Mater Manuf Eng., 2008, 26,11-17.

53. PETROBRÁS, 2017. Disponível em: http://sites.petrobras.com.br/minisite/assistenc iatecnica/public/downloads/manual_tecnico_di esels10_assistencia_tecnica_petrobras.pdf> acesso em 20/10/2017.

54. Pires, A \& Martinho, G. Life cycle assessment of a waste lubricant oil. management system. International Journal of Life Cycle Assessment,2012, 18.

55. Priola, A., Farrore, V., Arrighetti, $S$ \& MANCINI, G. Heterogeneous cationic catalytic systems suitable for the olegomerization of linear non-terminal olefins and a process for preparing said oligomers using said system. European patent 0068554 B1,1985.

56. Povedano, M. M. D \&Castro, M.D. Ultrasoundassisted extraction and in situ derivatization. Journal of Chromatography.2013, 226-234.

57. Rasberger, M. Oxidative degradation and stabilization of mineral oil based lubricants. Chemistry and Technology of Lubricants, 1997, 98-143.

58. Rincon, J., Canizares, P \& Garcia, T. M. Regeneration of Used Lubricant Oil by Polar Solvent Extraction. Ind. Eng. Chem. Res, 2005, 44, 4373-4379.

59. Rocher, V., Azimi, S., Moilleron, R \& Chebbo, G. Sources, distribution and variability of hydrocarbons and metals in atmospheric deposition in an urban area. Science of the Total Environment,2004, 337, 223-229.

60. Salem, S., Salem, A \& Babaei, A. A. Application of Iranian nano-porous $\mathrm{Ca}$ bentonite for recovery of waste lubricant oil by distillation and adsorption techniques. Journal of Industrial and Engineering Chemistry,2015, 23, 154-162.

61. Santos, O. C. J., Almeida, A. R., Carvalho, C. N. W. M., Conceição, M. M., Pequeno, G. M\&Souza, G. A. Avaliação da estabilidade térmica e oxidativa de óleo lubrificante automotivo recuperado usando o solvente metil-etil-cetona. 5 ${ }^{\mathrm{a}}$ Congresso Norte Nordeste de Química,2013.

62. Santos, J. C. O., Santos, I. M. G., Souza, A. G., Sobrinho, E. V., Fernandes \&Junior, V. J. Thermoanalytical and rheological characterization of automotive mineral lubricants after thermal degradation. Fuel,2004, 83, 2393-2399.

63. Santos, E. H. Síntese e Caracterização de Biolubrificantes a partir do óleo de soja refinado.Trabalho de Conclusão de Curso de graduação apresentado a Universidade Tecnológica Federal do Paraná, Curitiba,2011.

64. Senapati, S. K., Jaiswal, A. K., Mooken, R. T, Mishra, A. K, Srivastava, S. P e Bhatnagar, A. K. A new quality compressor oil with anti-wear 
performance. Proceedings of the $2^{\text {nd }}$ International Symposium on Fuels and Lubricant (ISFL),2000, 143-148.

65. Shiung, L. S; Russell, A. D \& Chase, H. A. Microwave pyrolysis, a novel process for recycling waste automotive engine oil. Energy,2010, 35, 2985-2991.

66. Silveira, E. L. C. et al. Determinação de contaminantes em óleos lubrificantes usados e em esgotos contaminados por esses lubrificantes. Quím. Nova, 2006, 29,11931197.

67. Silveira, E. L. C. et al. Determinação de metais em óleos lubrificantes, provenientes de motores de ônibus urbano, utilizando a FAAS. Quím. Nova, v. 33, n. 9, p. 18631867, 2010.

68. Simepetro. Agência Nacional do Petróleo. Disponível em<http://www.simepetro.com.br/wpcontent/u ploads/2009/05/portaria_130.pdf> Acesso em 27 out. 2017.

69. Singh, M. P., Ali, N., Tyagi, B. R., Srivastava S. P \& Bhatnagar A. K. Biodegradable lubricants. Proceedings of the 1st International Symposium on Fuels and Lubricants,1997, 369-374.

70. Song, G.,Seo, Y.,Pudasainee\& D., Kim, I. Characteristics of gas and residues produced from electric arc pyrolysis of waste lubricating oil. Waste Management, 2010, 30, 1230 1237.

71. Souza, O. F. Desenvolvimento de método de extração-floculação por aplicação de ondas ultrassônicas em óleo lubrificante usado. Dissertação de Mestrado, PROGRAMA DE PÓS-GRADUAÇÃO EM ENERGIA - PPGEN UNIVERSIDADE FEDERAL DO ESPÍRITO SANTO, 2015.

72. Srivastava, S. P. Advances in Lubricant Additives and Tribology. New Delhi: Tech Books International,2009, 365-368.

73. Srivastava, S. P. Developments in Lubricant Technology. Lubricant additives and their evaluation, 2014, $1^{\text {a }}$ ed., cap. 5, p.61.

74. Srivastava, S. P. Developments in Lubricant Technology. Rerefining and recycling of used lubricating oil,2014, $1^{\mathrm{a}}$ ed., cap. 18, p. 299.

75. Suen, Y. F., Ward, J \& Miller, T. Lubricating composition containing multifunctional borated hydroxylated amine salt of a hindered phenolic acid. US patent 8,334,242,2012.

76. Tagawa, K., Shimomura, Y., Sawada, K., Takigawa, K., Yoshida, T., Mitsumoto, S., Akiyama, E., Shibata, J., Suda, S., Yokota, H., Hata, H.,Hoshino, H., Nakao, H \& Konishl, S. Compressor oil composition, US patent 8,299,006, 2012.

77. Tatsumi, Y., Umehara, $K$ \& Lino, $S$ Antioxidant composition and lubricating oil composition containing same. US patent 2013,0184,190,2013.

78. Thoenes, H. W. Safety aspect for selection and testing of air compressor lubricants. Lubr Eng, 1975, 25, 409-411.

79. Tsai, W. T. An analysis of used lubricant recycling, energy utilization and its environmental benefit in Taiwan. Energy,2011, 36, 4333-4338.

80. Wagner, $H$ \&Luther, R. Mang, L. Lubricant base fluids based on renewable raw materials their catalytic manufacture and modification. Elsevier,2001, 429-442.

81. Vazquez-duhalt, R. Environmental impact of used motor oils. Science Total Environment, 1989, 79.

82. Willing, A. Lubricants based on renewable resources - an environmentally compatible alternative to mineral oil product. Chemosphere, 2001, 43, 89-98.

83. Yao, T. et al. The effect of environmental factors on the adsorption of lubricating oil onto expanded graphite.Journal of Molecular Liquids, 2016, 218, 611-614.

84. Yang, $H$. et al. Effect of water chemistry on the adsorption of lubricating oil on oxidized graphite. Journal of Molecular Liquids,2016, 219, 1157-1160.

85. YIN, T.,ZHANG, X.,LIU, X\&WANG, C.Resource recovery of Eichhornia crassipes as oil superabsorbent. Marine Pollution Bulletin,2017, 118, 267-274.

86. Zakarian, J. A., Robson, R. J \& Farrell, T. R. All-hydroprocessing route for high-viscosity index lubes. Energy Prog,1987, 7, 59-64.

87. Zaky, T. \& Tawfik, M. S. Production of lubricating base oil from slop wax by different subsequent refining techniques. Fuel Processing Technology,2011, 92, 447-451.

88. Zhang, H., Tang, J., Wang, L., Liu, J., Guray, G. R \& Sun, K. A novel bioremediation strategy for petroleum hydrocarbon pollutants using salt tolerant Corynebacterium variabile HRJ4 and biochar. Journal of Environmental Sciences, 2016, 47, 7-13.

89. Zubaidy, E. A. H \& Abouelnasr, D. M. Fuel recovery from waste oily sludge using solvent extraction. Process Safety and Environmental Protection, 2010, 88, 318-326.

90. Zzeyani, S., Mikou, M., Naja, J \& Elachhab, A.Spectroscopic analysis of synthetic lubricating oil. Tribology International, 2017, 114,27-32. 
Tabela 1 -Tipos de solventes, proporção solvente/óleo, rendimento do óleo base em porcentagem e seus respectivos materiais adsorventes.

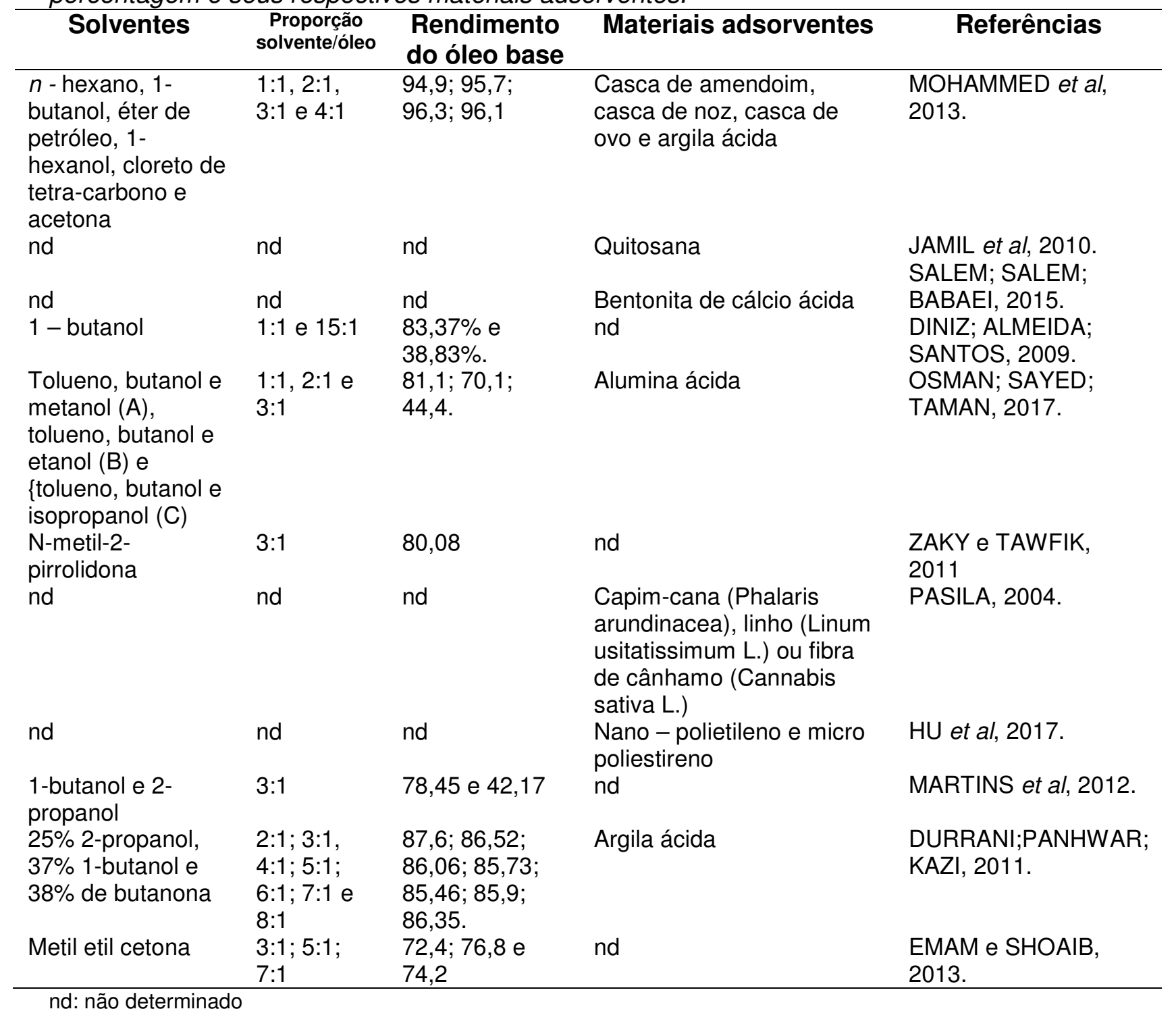

nd: não determinado

Fonte:autoria própria 
Tabela 2.Características dos óleos básicos recuperados.

\begin{tabular}{|c|c|c|c|c|c|}
\hline Cor & Visc. $40^{\circ} \mathrm{C}$ & Visc. $100^{\circ} \mathrm{C}$ & \%Cinzas & $\begin{array}{l}\text { Classificação } \\
\text { ANP, } 1999 .\end{array}$ & Referências \\
\hline 0,$12 ; 0,25 ; 0,15$ & $85,80,75$. & 11,$1 ; 9,8 ; 10,7$ & nd & $\begin{array}{l}\text { Neutro pesado } \\
\text { RR }\end{array}$ & $\begin{array}{l}\text { MOHAMMED } \\
\text { et al, } 2013 .\end{array}$ \\
\hline nd & nd & nd & nd & nd & $\begin{array}{l}\text { JAMIL et al, } \\
2010 \text {. }\end{array}$ \\
\hline nd & 46,5 & 7,4 & nd & $\begin{array}{l}\text { Neutro médio } \\
\text { RR }\end{array}$ & $\begin{array}{l}\text { SALEM; } \\
\text { SALEM; } \\
\text { BABAEI, } 2015 .\end{array}$ \\
\hline nd & nd & nd & nd & nd & $\begin{array}{l}\text { DINIZ; } \\
\text { ALMEIDA; } \\
\text { SANTOS, } \\
2009 .\end{array}$ \\
\hline nd & nd & nd & nd & nd & $\begin{array}{l}\text { OSMAN; } \\
\text { SAYED; } \\
\text { TAMAN, } 2017 .\end{array}$ \\
\hline \multirow[t]{2}{*}{ nd } & $\begin{array}{l}36,08 \\
33,49 \\
30,49\end{array}$ & $\begin{array}{l}5,75 ; 5,53 \\
5,28\end{array}$ & $\begin{array}{l}33,66 \\
34,69 \\
41,88\end{array}$ & Neutro leve & $\begin{array}{lr}\text { ZAKY } & e \\
\text { TAWFIK, } 2011\end{array}$ \\
\hline & & & & & PASILA, 2004. \\
\hline nd & nd & nd & nd & nd & HU et al, 2017. \\
\hline nd & nd & nd & nd & nd & $\begin{array}{l}\text { MARTINS et } \\
\text { al, 2012. }\end{array}$ \\
\hline nd & nd & nd & nd & nd & $\begin{array}{l}\text { DURRANI; } \\
\text { PANHWAR } \\
\text { KAZI, } 2011 .\end{array}$ \\
\hline 6 & 75,6 & 9,9 & nd & $\begin{array}{c}\text { Neutro pesado } \\
\text { RR }\end{array}$ & $\begin{array}{l}\text { EMAM e } \\
\text { SHOAIB, } \\
2013 .\end{array}$ \\
\hline
\end{tabular}

Tabela 3: Técnicas de caracterização dos lubrificantes.

\begin{tabular}{ll}
\hline Caracterização dos lubrificantes & Referências \\
\hline nd & MOHAMMED et al, 2013. \\
nd & JAMIL et al, 2010. \\
nd & SALEM; SALEM; BABAEI, 2015 \\
FTIR e TGA/DTG & DINIZ; ALMEIDA; SANTOS, 2009. \\
nd & OSMAN; SAYED; TAMAN, 2017. \\
nd & ZAKY e TAWFIK, 2011 \\
nd & PASILA, 2004. \\
nd & HU et al, 2017. \\
nd & MARTINS et al, 2012. \\
nd & DURRANI;PANHWAR; KAZI, 2011. \\
nd & EMAM e SHOAIB, 2013. \\
\hline nd: não determinado &
\end{tabular}

Fonte:autoria própria 
Tabela 4:Representação das bandas características do óleo novo, OLUC e óleo recuperado e suas respectivas atribuições.

\begin{tabular}{ll}
\hline Número de onda $\left(\mathbf{c m}^{-1}\right)$ & Atribuição \\
\hline 723,821 e 1158 & Deformação C-H para $\mathrm{CH}_{2}{\mathrm{e} \mathrm{CH}_{3}}$ \\
1378 e 1470 & Compostos orgânicos oxigenados \\
1600 & C-C \\
1705 & C-O
\end{tabular}

nd: não determinado

Fonte:autoria própria

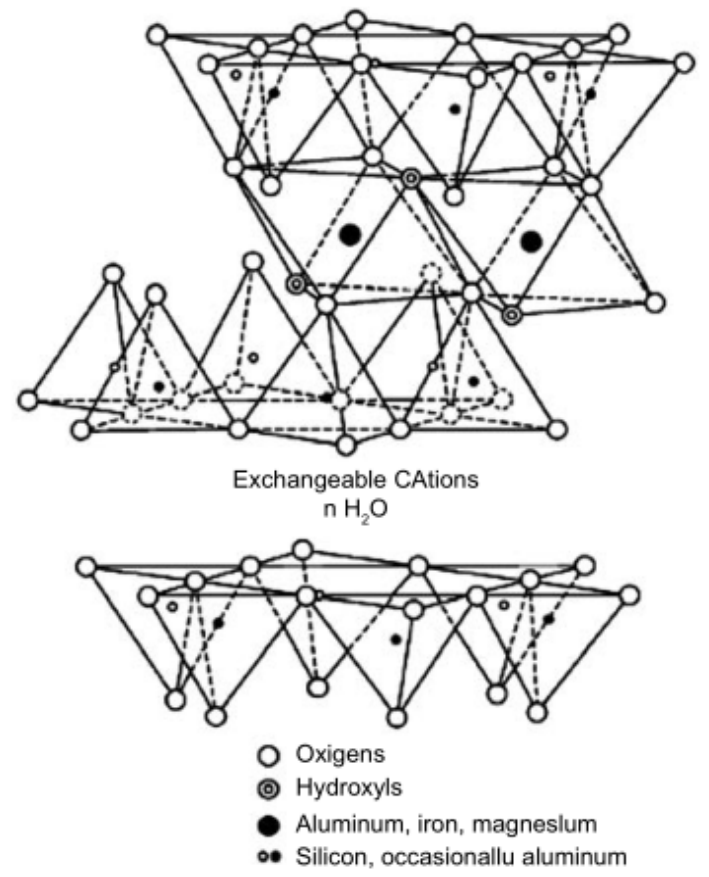

Figura 1. Representação da fórmula estrutural da esmectita.

Fonte:PATRICIO; HOTZA e JÚNIOR, 2014.

PERIÓDICO TCHÊ QUÍMICA •www.periodico.tchequimica.com• Vol. 15 N. 30.

• ISSN 1806-0374 (impresso) • ISSN 1806-9827 (CD-ROM) • ISSN 2179-0302 (meio eletrônico)

(C) 2018. Porto Alegre, RS. Brasil

The Periódico Tchê Química (ISSN: 1806-0374; 2179-0302) is an open-access journal since 2004. Journal DOI: 10.52571/PTQ. http://www.tchequimica.com. This text was introduced in this file in 2021 for compliance reasons.

(c) The Author(s)

OPEN ACCESS. This article is licensed under a Creative Commons Attribution 4.0 (CC BY 4.0) International License, which permits use, sharing, adaptation, distribution, and reproduction in any medium or format, as long as you give appropriate credit to the original author(s) and the source, provide a link to the Creative Commons license, and indicate if changes were made. The images or other third-party material in this article are included in the article 's Creative Commons license unless indicated otherwise in a credit line to the material. If material is not included in the article's Creative Commons license and your intended use is not permitted by statutory regulation or exceeds the permitted use, you will need to obtain permission directly from the copyright holder. To view a copy of this license, visit http://creativecommons.org/licenses/by/4.0/. 